\title{
Ensuring Better Service Assurance using Scalable Monitoring and Resource Management
}

\author{
Mohammed J. Mohammed \\ College of Engineering Nawroz University, Duhok, Kurdistan Region - Iraq
}

\begin{abstract}
This research is about the construction of scalable monitoring system for monitoring the network in both offline and online mode. Continuous monitoring of network status and its resources like host, router etc in a scalable manner to ensure the proper operation of the network is the goal of this proposed system. The proposed system is scalable in terms of network size, speed and the number of customers subscribed to value-added services. It also provides the measurements for network provision, dynamic resource allocation, route management and the in-service service verification.
\end{abstract}

KEYWORDS: IP, Monitoring, Traffic Engineering, Differentiated Services, Active/Passive Measurements, Scalability.

\section{INTRODUCTION}

Quality of Service (QoS) monitoring becoming crucial role for providing quantified QoS based services and assurance to the Internet Service Providers (ISP). In this research, the performance of a customer's requested service requirements are explained in a Service Level Specification (SLS) [1]. SLS is the technical part of a Service Level Agreement (SLA) with the provider, and in such a provider's network, Quality of Service is affirmed in a scalable manner with the differentiated services where routers are used to aggregate the network traffic that belongs to several service classes as per the predefined QoS policies. Quality of Service (QoS) can be defined as the service offered where one or more performance measures are quantified like throughput, delay, loss, delay variation etc as the network attempts to offer different service categories like real-time traffic, virtual private networks and best effort services, network and service monitoring are all important for providing end to end QoS services and assurance. In this, monitoring activity is not only has

Academic Journal of Nawroz University (AJNU)

Volume 7, No 3 (2018).

Received 17 Feb 2018;

Regular research paper : Published 20 June 2018

Corresponding author's e-mail : Mjm562000@yahoo.com

Copyright (C2018 Mohammed J. Mohammed.

This is an open access article distributed under the Creative

Commons Attribution License.

the diagnostic role, but becomes an important tool for supporting network operations and also providing service auditing. Given the masses of services with different performance measurements, the information for measurement needs to be gathered at finer granularity than that of per ingress-egress node pairing and also the service type needs to be taken into consideration. So the design and implementation of scalable monitoring system should provide network provision measurements, dynamic resource allocation, route management and in-service verification for value added services [2]. The goal of the monitoring system is not only to measure the quality of service metrics, but to provide information in order to guarantee the contracted services also by means of tuning and controlling the network resources. A monitoring system provides information for the following categories

1. Help dynamic online traffic engineering (TE) in making provisioning decisions for optimizing the usage of network resources according to short to medium term changes and this information can be used to take appropriate activities on setting new routes, modifying existing routes, performing load balancing and rerouting traffics.

2. Help offline TE in providing analytical traffic and information about performance for long term planning in order to optimize the network usage and to avoid undesirable conditions.

3. Verify whether the quality of service performance guarantees committed to in service level specifications are in fact being met and SLSs can differ depends on the types of services offered and different types of SLS have different quality of service requirements that needs 
processing of different information types and it is required per service type for verification of in-service traffic and service performance characteristics. Monitoring can be occurred at different abstraction levels. For deriving packet, application, user/customer, traffic aggregate, node and network wide levels information, measurements can be used. There are two methods: active and passive measurements; can be used to perform low level measurements in monitoring the systems. Active measurements will inject synthetic traffic into the network based on scheduled sampling in order to observe network performance and the active measurement tools require co-operation from both measurement end-points. Passive measurement will be mainly used for the purpose of observing actual traffic patterns in the network. And it can also be used to observe the network performance monitoring. To conduct traffic monitoring, continuous gathering of data and monitoring of links at full load is also needed. Three aspects are there in the scalability in QoS enabled IP networks and their associated services and they are: network topology size, granularity and number of classes of service supported, and the subscribed customer number. The network topologies are characterized by a number of parameters like number of nodes and links, logical and physical connectivity degree and the network diameter. A large number of subscribed customers badly require huge amount of valuable information and it is to be gathered for the monitoring of service and service assurance, as in service verification of QoS performance and its guarantees are required for every individual customers. The monitoring system scalability is the ability of effectively positioning the system at the scale of a large network that offers a number of services to the large number of customers. The monitoring systems have a number of design features for a wide range of monitoring tasks which ensure a scalable solution for providing the expected performance outcome. The monitoring task contains data/information collection, data aggregation and data analysis for providing necessary feedback.

\section{MATERIALS AND TECHNOLOGY}

The scalable monitoring systems principles include the following.

1.At the aggregated level, define the QoS monitoring process granularity.

2. At the node level, disperse the data collection system measurement.

3.Process data close to the source to minimize the information exchange.

4.Substitute Edge - to - Edge by Hop - by - Hop measurements.

The scalable monitoring system is implemented by using the Simple Network Management Protocol (SNMP) [5] and it is used to communicate management information between the network management stations and the agents in the network elements. SNMP is an application layer protocol for the exchange of management information between the network devices. The major operations supported by Simple Network Management Protocol are: GETREQUEST, GETNEXTREQUEST, SETREQUEST, TRAP and GETRESPONSE. The current status of the network is monitored by the SNMP protocol is accessed by the Management Information Base (MIB) of the network resources like host, routers, switches, bridges and so on. The Management Information Base also contains all network related information like interfaces, system information, IP information etc.

\section{WORKING}

The scalable monitoring system contains the following elements: Node Monitor, Network Monitor and SLS Monitor. Node Monitor is responsible for the node related measurements, Network Monitor is responsible for network related post-processing of measurement of data using statistical functions and SLS Monitor is responsible for customer related service monitoring, auditing and reporting. Repository Monitoring contains data store for storing large amount of data and information store for storing small amount of data (configuration type information) and Monitoring Graphical User Interface (GUI) is used to display the results of measurements. The scalable monitoring system works as follows and the system consists of the following phases:

Configuration: Monitoring first registers to one or more of the monitoring components like node, network or SLS are required by the clients. The client must request monitoring actions by providing the necessary information. For example like metric to be monitored, sampling, summarization periods, thresholds etc.

Execution: NodeMons performs the necessary measurement activity based on the received configuration and the passive measurements will be performed by using SNMP.

Reporting and Exception: The analyzed data and push the threshold crossing events to the interested monitoring clients are sent back by NodeMons. Current and historical long-term in-depth statistical analysis of monitoring data are requested by clients are provided by the network and SLS monitoring. System administrator will request the graphical display of any measurement of data at node, network and SLS levels.

\section{COMPARISON WITH EXISTING SYSTEM}

The earlier network monitoring system makes use of Traffic Engineering (TE) algorithm, and it can be defined as a set of techniques to maximize the utilization of network resources [3].This algorithm mainly deals with the performance optimization of operational IP networks and encompasses the application of principles to the measurement, characterization and control of traffic. The 
following drawbacks are there for the existing TE method of monitoring and they are

1.An overview of the network status for their dynamic reactions needed by TE algorithm.

2.The optimization objectives may change over time as new requirements and policies are imposed since TE is a continual and iterative process of network performance.

Therefore, we are going with the proposed scalable monitoring system which is generic enough to adjust with the so called changes as well as it does not require knowledge of the history for taking actions. The proposed monitoring system is scalable and also dynamic in nature, and it reflects the current state of the network based on the real time data.

\section{CONCLUSION}

Careful network engineering and its traffic are necessary for the resource usage efficiency while meeting the required performance target when delivering quality of service based value added IP services. The proposed system is distributed for guarantee quick response times and minimizes the necessary management traffic. The proposed scalable monitoring system allows for good accuracy for one-way delay and packet loss and it also provides highly comparable edge-to-edge and hop-byhop results. The proposed principles result in the scalable monitoring systems that can be contribute towards operationally optimized traffic networks which can support a large number of customers.

\section{REFERENCES}

1. D. Goderis et al., "Service Level Specification Semantics and Parameters," Internet draft, draft-tequilasls-02.txt.

2. S. Blake, D. Black, et al., "An Architecture for Differentiated Services", Informational RFC-2475.

3. A. Feldman et al., "Netscape: Traffic Engineering for IP Networks", IEEE Network Magazine, Vol. 14, No. 2, pp. 11-19, March/April 2000.

4. "A Scalable Real-time Monitoring System for Supporting Traffic Engineering" Abolghasem Asgari, Panos Trimintzios, Mark Irons, George Pavlou, Richard Egan, Steven V. den Berghe. www.snmplink.org.

\section{Figure (1) : Architecture of Scalable Monitoring System}

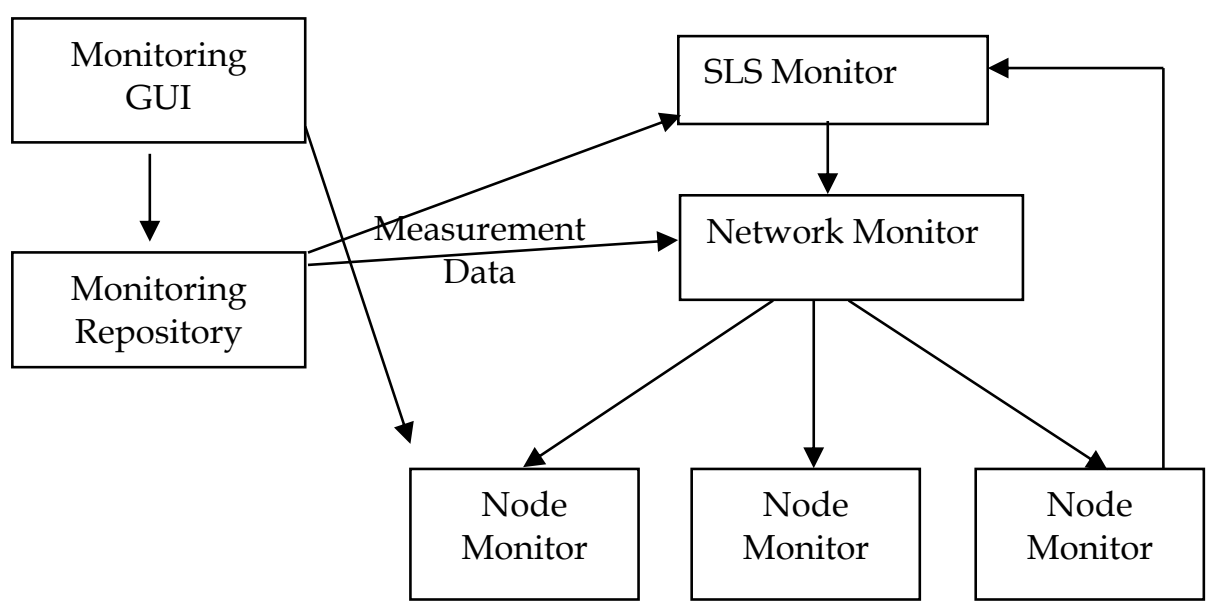

